\title{
Multi-user Communication Based OFDM-UWB System under Gaussian and Non-Gaussian Noisy Channel
}

\author{
Doaa E. El. Matary ${ }^{*}$, Esam A. A. Hagras, Hala Mansour Abdel-Kader \\ Communication and Electronics Department, Faculty of Engineering, Shoubra, Benha University, Cairo, Egypt \\ *Corresponding author: doaa_elmatary@yahoo.com
}

\begin{abstract}
For a multiuser Ultra Wide Band (UWB) system, the performance is mainly limited by inherent noise and interferences such as Multiple Access Interference (MAI) and Inter Symbol Interference (ISI) which are poorly approximated by a Gaussian distribution because of their impulsive nature. A combination between Orthogonal Frequency Division Multiplexing (OFDM) and Interleave Division Multiple Access (IDMA) has been considered as an efficient technique due to its bandwidth efficiency, and robustness against MAI and ISI. Moreover, OFDMIDMA technique has the ability to allow frequency diversity and performance improvement with the help of efficient FEC coding technique such as Low Density Parity Check (LDPC) code. Therefore, this paper addresses the performance of LDPC-coded-IDMA-based OFDM-UWB communication system under Gaussian and non-Gaussian noisy channels. Three different models as Symmetric Alpha Stable (S $\alpha$ S), Laplacian model and Gaussian Mixture Model (GMM), have been introduced for approximating the non-Gaussian noise which are more realistic for UWB system. Simulation results show that LDPC-coded OFDM-IDMA system is robust against noise and interferences in UWB indoor environment, and achieves good performance under Cauchy $(\mathrm{S} \alpha \mathrm{S})$ model rather than other nonGaussian models with simple Chip-By-Chip (CBC) iterative receiver. Furthermore, the proposed system gains a significant performance improvement compared with the un-coded OFDM-IDMA-UWB system under the studied noise models.
\end{abstract}

\section{Keywords: UWB, OFDM-IDMA, LDPC, GMM, Laplace, S $\alpha$ S, CBC}

Cite This Article: Doaa E. El. Matary, Esam A. A. Hagras, and Hala Mansour Abdel-Kader, "Multi-user Communication Based OFDM-UWB System under Gaussian and Non-Gaussian Noisy Channel." American Journal of Electrical and Electronic Engineering, vol. 5, no. 4 (2017): 136-143. doi: 10.12691/ajeee-5-4-3.

\section{Introduction}

Recently, multicarrier transmission for UWB based OFDM for short-range high-rate wireless systems that have attracted a great interest [1] due to inherent advantages of high speed transmission, immunity towards multipath, low power consumption, high spectral efficiency, and improved channel capacity [2,3]. For a multiuser UWB system, the performance is mainly limited by inherent noise and interferences such as MAI and ISI [4].

Indoor environments are subjected to impulsive (non-Gaussian) noise because of the wide spread of electronic devices. MAI in UWB radio system is poorly approximated by a Gaussian distribution since it is an impulse-like. Therefore, Gaussian approximation is not fully efficient for UWB systems. Several alternative distributions for approximating impulsive noise in UWB systems are motivated and compared [4,5]. These distributions have in common that they are more impulsive than Gaussian approximation, with a greater area in the tails of the probability density function (pdf) compared to a Gaussian pdf [5].

Interleave Division Multiple Access (IDMA) is a recently proposed spread spectrum multiple access scheme, user distinct interleavers are the unique feature to distinguish users for IDMA system [6]. Chip interleaving was first introduced in [6] to mitigate the burst impulsive noise disturbance. The user separation relies on iterative multiuser detection [7]. Moreover, IDMA features are: suitable for wide or narrow band transmission, low receiver cost, high power and spectral efficiency, diversity against fading and flexible rate adaptation [7]. One important feature of IDMA-UWB system is to mitigate the MAI and multipath fading effect depending on the turbo type iterative detection strategy [8].

The OFDM technique has received a lot of interest; because it allows a high spectrally efficient transmission over frequency selective broadband channels at reasonable cost of implementation. The main idea behind OFDM is to split the input data in many sub-channels and transmit each one of them in a different sub-carrier with a low transmission rate, with sub-carriers chosen such that they are mutually orthogonal. Each sub-channel experiments a narrow band channel making the receiver structure less complex [9]. However, OFDM is not able to handle the burst errors caused by impulsive noise in multipath channel.

Recent papers investigate the combination of OFDM and IDMA to avoid their individual disadvantages $[9,10]$. The results prove that OFDM-IDMA system inherits so many advantages over other alternatives such as robustness against MAI and ISI, and ability to handle the whole burst 
errors caused by impulsive noise in multipath channel. Moreover, OFDM-IDMA system has the ability to allow frequency diversity with the help of efficient FEC coding technique.

LDPC codes are considered as one of the most powerful FEC techniques due to their superior error correction capability with simple iterative decoding. Hence, these codes have been utilized in most of current UWB systems $[11,12]$. LDPC coding technique is used to reduce the effect of MAI in multiuser UWB system which leads to performance improvement [11]. Moreover, LDPC codes achieve better gains in system capacity with lower complexity [12]. The integration of multiple access and coding techniques are used to improve the performance greatly. Therefore, adopting LDPC code in an IDMA-based system is efficient since both MUD in IDMA layer and LDPC require computing Logarithm Likelihood Ratio (LLR) values [13]. So, LDPC coding technique has been combined with IDMA scheme based OFDM system to overcome the multipath channel imperfections efficiently and improve the performance as described in [13]. More advantages of LDPC OFDM-IDMA have been introduced in literature [13].

In this paper, a comparative study is held between the proposed LDPC-coded OFDM-IDMA-UWB and un-coded OFDM-IDMA UWB systems to clarify the performance improvement under the studied Gaussian and non-Gaussian noise models.

This paper is organized as follows: section II presents the UWB channel and noise models, section III describes the LDPC-coded OFDM-IDMA-UWB transmitter and receiver structures, Elementary Signal Estimator (ESE), and Decoder (DEC) functions. The simulation results of the proposed system are introduced in Section IV, and finally section V concludes the paper.

\section{UWB Channel and Noise Models}

The design of any communication system depends on studying the characteristics of the channel and noise nature. Indoor UWB environments are subjected to multipath fading and impulsive noise which have a strong effect on the system performance assessment. Hence, the accurate modeling of channel and noise is a very important issue. Such models create the facility for calculation of large and small-scale characteristics which are necessary for efficient system design.

\subsection{UWB Channel Model}

The standardized channel model for indoor UWB environments has been proposed by the channel modeling subcommittee of the IEEE 802.15.3a Task Group. Such model is a modified version of the Saleh-Valenzuela (S-V) model, where the Rayleigh distribution of the channel coefficient amplitude is replaced by the log-normal distribution [14]. Four different channel models (CM1, CM2, CM3, and CM4) based on the average distance between transmitter and receiver, as well as the propagation conditions were specified by the IEEE 802.15.3a subcommittee [14].

\subsection{Impulsive Noise Model}

The indoor noise is typically impulsive in nature due to the interference emanating from other electronic devices. Impulsive noise is typically characterized as noise whose distribution has an associated "heavy tail" behavior. That is, their pdf approaches zero more slowly than a Gaussian pdf. The noise in UWB channel can be modeled as [15]:

$$
n(j)=n_{a}(j)+n_{I}(j)
$$

Where $n(j)$ is the noise, which can be divided into $n_{a}(j)$ which is background (AWGN) noise with zero mean and variance $\sigma^{2}$, and $n_{I}(j)$ which is the Impulsive noise with zero mean and variance $\sigma_{I}^{2}$. Impulsive noise $n_{I}$ can be accurately modeled by three different models, symmetric alpha stable, Laplacian model, Gaussian mixture model, which will be introduced below.

\subsubsection{The Symmetric Alpha Stable Model}

According to [16], the Symmetric Alpha Stable $(\mathrm{S} \propto \mathrm{S})$ distribution can be completely determined by two parameters: (1) a characteristic exponent $\propto \in[0,2]$, which indicates the characteristic of the tail of the $S \propto S$ distribution; (2) a divergence (or scale) parameter $\gamma \in(0, \infty)$ which is analogous to the variance of the Gaussian distribution. One difficulty of the $S \propto S$ distribution is that they have no closed-form expressions for their pdf $f_{\alpha}(x)$ except for $\propto=1$ (i.e., the Cauchy distribution) and $\propto=2$ (i.e., the Gaussian distribution). The pdf at $\alpha=1$ (Cauchy detector) can be defined as [17]:

$$
\mathrm{f}_{\text {Cauchy }}(\mathrm{r})=\frac{\gamma}{\pi} \frac{1}{\gamma^{2}+(\mathrm{r}-\mu)^{2}}
$$

Where $\mu$ is the location parameter of the distribution.

\subsubsection{The Laplacian Model}

The pdf of Laplacian noise is given by [18]:

$$
\mathrm{f}_{\text {Laplace }}(\mathrm{r})=\frac{1}{2 \mathrm{C}} \exp \left(-\frac{|\mathrm{r}-\mu|}{\mathrm{C}}\right),-\infty<x<\infty
$$

Where $\mu$ is the location parameter (mean), and $C$ is the scale parameter ( $2 \mathrm{C}^{2}=$ variance) and its value is always positive. By varying the scale parameter $\mathrm{C}$, different tail behaviors can be obtained. The higher the value of $\mathrm{C}$ is, the more slowly the tail decays when the location parameter is constant.

\subsubsection{The Gaussian Mixture Model}

A simple two term mixture pdf is given by $[19,20]$ :

$$
\begin{aligned}
& \mathrm{f}_{\mathrm{GMM}}(\mathrm{r})=(1-\epsilon) \mathrm{g}(\mathrm{r})+\epsilon \mathrm{h}(\mathrm{r}) \\
& =\frac{(1-\epsilon)}{\sqrt{2 \pi \sigma_{\mathrm{g}}^{2}}} \mathrm{e}^{-\frac{(\mathrm{r}-\mu)^{2}}{2 \sigma_{\mathrm{g}}^{2}}}+\frac{\epsilon}{\sqrt{2 \pi \sigma_{\mathrm{h}}^{2}}} \mathrm{e}^{-\frac{(\mathrm{r}-\mu)^{2}}{2 \sigma_{\mathrm{h}}^{2}}}
\end{aligned}
$$

Where $\mathrm{g}($.$) is the nominal Gaussian pdf with variance \sigma_{\mathrm{g}}{ }^{2}$ and $h($.$) is the heavier tailed Gaussian with$ variance $\sigma_{\mathrm{h}}{ }^{2}=\eta \sigma_{\mathrm{g}}{ }^{2}$, where $\eta \geq 1$, is the impulsive part's 
relative variance with respect to (w.r.to) nominal Gaussian noise variance (also called Noise Variance Ratio NVR). The parameter $\epsilon \in[0,1]$ controls the contribution of impulsive component to the whole pdf.

\section{LDPC-Coded-IDMA-OFDM-UWB Transmitter and Receiver Structures}

The transmitter and receiver structures of the proposed LDPC-coded-OFDM-IDMA-UWB system for multi-user communication scenario are depicted in Figure 1. For simplicity, synchronous BPSK signaling is considered over time in-variant multipath channel.

\subsection{The Transmitter Structure}

For the user-k, the information data sequence $d_{k} \in$ $\{0,1\}$ is encoded using FEC LDPC encoder into a coded sequence $c_{k}$. The $n^{\text {th }}$ coded bit of user-k, $c_{k}(n), n=$ $1,2, \ldots, N$, is spread using a balanced spread sequence $S$. The same spreading code is applied to all users $S_{k} \in$ $\{+1,-1\}$. The obtained chip sequence is written as $\left\{c_{k}(j), j=1,2, \ldots, J\right\}$, where $J=N \times S$ is the chip length. A specific distinct chip level random interleaver $\left\{\pi_{\mathrm{k}}, \mathrm{k}=\right.$ $1,2, \ldots, \mathrm{K}\}$ is employed for user separation [21].

The interleaved chip sequences are mapped using BPSK onto the modulated symbols $\left\{\mathrm{x}_{\mathrm{k}}(\mathrm{j}), \mathrm{j}=1,2, \ldots, \mathrm{J}\right\}$ which are the elements of BPSK constellation. For simplicity, the symbol mapping process is not shown in Figure 1.
The BPSK symbols $\mathrm{x}_{\mathrm{k}}(\mathrm{j})$, considered an input to OFDM stage, are transmitted through orthogonal subcarriers by using Inverse Fast Fourier Transform (IFFT) operation, where the total number of subcarriers for OFDM transmission is denoted by $\mathrm{N}_{\mathrm{c}}$. In addition, a Cyclic Prefix CP of length $\mathrm{L}_{\mathrm{g}}$, assumed to be larger than the channel length, is inserted to prevent ISI and ICI [22]. As a result, the time-domain data sequence, including $\mathrm{CP}$, has the form:

$$
v_{k}=\frac{1}{N_{c}} \sum_{u=0}^{N_{\mathrm{C}}-1} \mathrm{x}_{\mathrm{k}, \mathrm{u}} \mathrm{e}^{\mathrm{i} 2 \pi \mathrm{ut} / \mathrm{N}_{\mathrm{C}}}, \mathrm{t}=-\mathrm{L}_{\mathrm{g}}, \ldots, 0, \ldots, \mathrm{N}_{\mathrm{C}}-1
$$

Where $x_{k, n}$ is the modulated data of the OFDM symbol of the user-k which is mapped to the $\mathrm{u}^{\text {th }}$ subcarrier. The OFDM signal $v_{k}$ is transmitted through the multipath channel. It is assumed that the multipath channel impulses of each user, $h_{k}=\left[h_{k}(0), h_{k}(1), \ldots, h_{k}(L-1)\right]$, are stationary in a frame period and mutually independent, where $\mathrm{L}$ denotes the number of resolvable paths. The output signal $r(j)$ is a linear superposition of independently symbols for each user. The output of multipath channel is written as [10]:

$$
\mathrm{r}(\mathrm{j})=\sum_{\mathrm{k}=1}^{\mathrm{K}} \mathrm{h}_{\mathrm{k}} * v_{\mathrm{k}}+\mathrm{n}(\mathrm{j})
$$

Where $*$ denotes the convolution, $\mathrm{h}_{\mathrm{k}}$ the UWB channel coefficient for user-k and $n(j)=n_{a}(j)+n_{I}(j)$ the total noise.

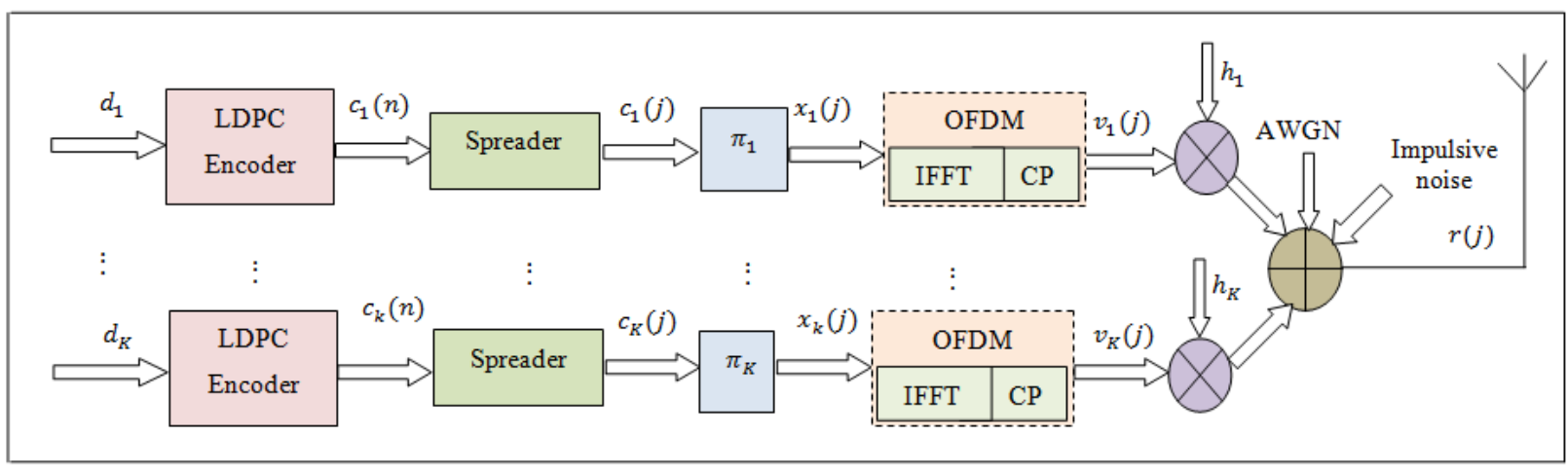

(a) Transmitter

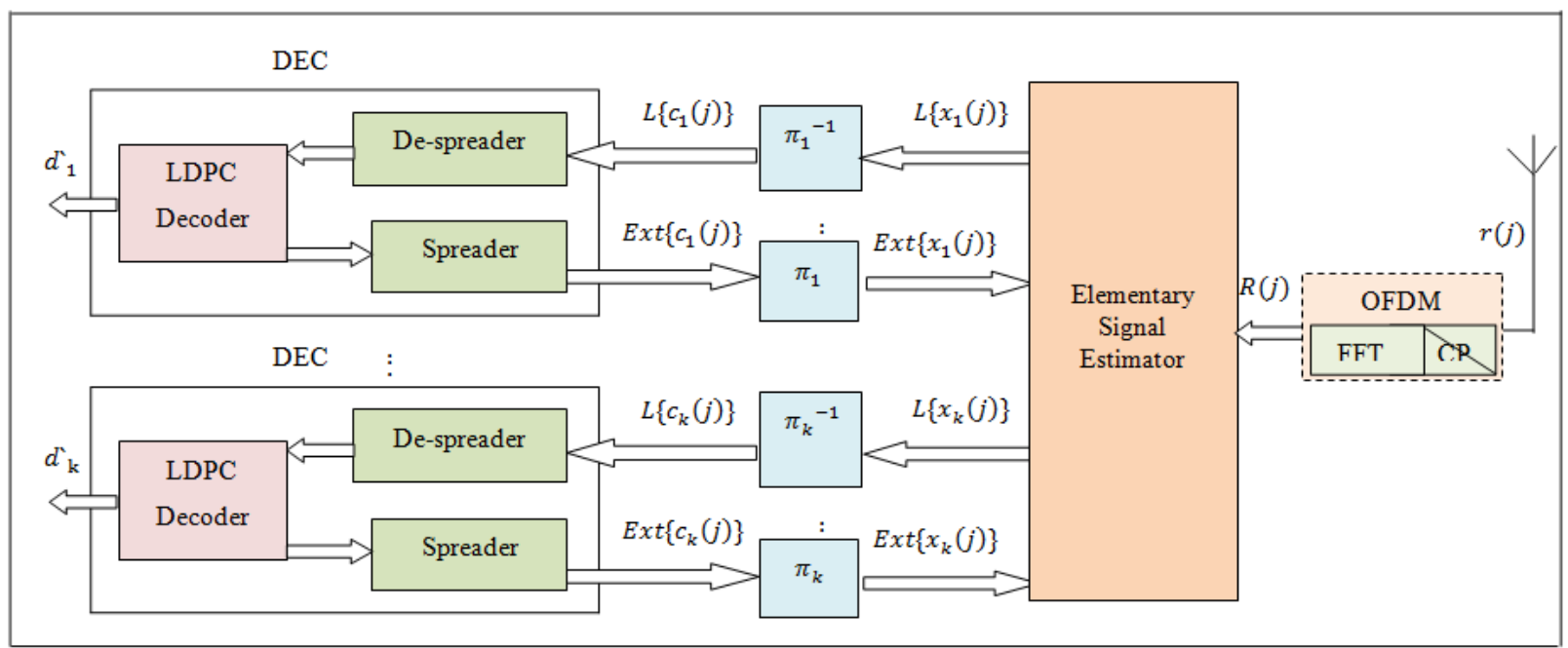

(b) Receiver 


\subsection{The Receiver Structure}

The turbo-type iterative receiver structure of the proposed system is depicted in Figure 1(b) which includes two main parts, Elementary Signal Estimator (ESE) and a bank of $k$ single user A Posteriori Probability (APP) decoders (DECs) [11]. A standard form of Sum Product Algorithms (SPA) is adopted for LDPC decoder due to its capacity to achieve desirable performance and complexity trade-offs. At the receiver side, OFDM demodulation is carried out before iterative MUD process [12]. The received signal in frequency domain after OFDM demodulation, CP removal and FFT operation, can be expressed as

$$
R(j)=\sum_{k=1}^{K} H_{k}(j) x_{k}(j)+N(j)
$$

Where $x_{k}(j)$ and $H_{k}(j)$ are respectively the data chip over the $\mathrm{u}^{\text {th }}$ subcarrier and the corresponding channel tap for the user-k estimated from $H_{k}(j)=\sum_{l=0}^{L-1} h_{k}(l) \cdot e^{-i 2 \pi l u / N_{c}}$. $N(j)$ is a FFT of $n(j)$ [23]. The received signal $R(j)$ is processed iteratively by means of ESE and DECs functions which discussed as follows.

\subsubsection{The ESE Function}

The ESE performs a coarse chip-by chip estimation. Re-write Eq. (7), the received signal at time instant $\mathrm{j}$ is:

$$
R(j)=\sum_{k=1}^{K} H_{k}(j) x_{k}(j)+\xi_{k}(j)
$$

Where $\xi_{\mathrm{k}}(\mathrm{j})=\sum_{\mathrm{m} \neq \mathrm{k}} \mathrm{H}_{\mathrm{m}}(\mathrm{j}) \mathrm{x}_{\mathrm{m}}(\mathrm{j})+\mathrm{N}(\mathrm{j})$ represents a distortion (including interference plus noise) with respect to user-k. It is assumed that the channel coefficients $\mathrm{H}_{k}$ are known a priori at the receiver. Moreover, it is supposed that the received signal is perfectly synchronized. A CBC iterative detection based on LLR of the received chip sequence approximated as either Gaussian or non-Gaussian noise.

In case of Gaussian approximation (i.e. $n(j)=$ $\mathrm{n}_{\mathrm{a}}(\mathrm{j})$ only), $\mathrm{x}_{\mathrm{k}}(\mathrm{j})$ is treated as a random variable with mean $E\left(x_{k}(j)\right)$ and variance $\operatorname{Var}\left(x_{k}(j)\right)$ (initialized to 0 and 1 respectively) [11]. Then from (8), we have:

$$
\begin{gathered}
E(R(j))=\sum_{k=1}^{K} H_{k}(j) E\left(x_{k}(j)\right) \\
\operatorname{Var}(R(j))=\sum_{k=1}^{K}\left|H_{k}(j)\right|^{2} \operatorname{Var}\left(x_{k}(j)\right)+\sigma^{2}
\end{gathered}
$$

Where $\sigma^{2}$ is the power of the background (AWGN) occurs during the $\mathrm{j}^{\text {th }}$ chip. Using the central limit theorem [23], $\xi_{\mathrm{k}}(\mathrm{j})$ in (8) can be approximated by a Gaussian random variable with:

$$
\begin{gathered}
E\left(\xi_{k}(j)\right)=E(R(j))-E\left(H_{k}(j) x_{k}(j)\right) \\
\operatorname{Var}\left(\xi_{k}(j)\right)=\operatorname{Var}(R(j))-\left|H_{k}(j)\right|^{2} \operatorname{Var}\left(x_{k}(j)\right)
\end{gathered}
$$

The ESE outputs are the (LLRs) about $\left\{\mathrm{x}_{\mathrm{k}}(\mathrm{j})\right\}$ computed based on (9) (using (10)) as [12]:

$$
\begin{aligned}
& L\left(x_{k}(j)\right) \equiv \log \left(\frac{\operatorname{Pr}\left(x_{k}(j)=+1 \mid R(j)\right)}{\operatorname{Pr}\left(x_{k}(j)=-1 \mid R(j)\right)}\right) \\
& =\exp \left(-\frac{\left(R(j)-E\left(\xi_{k}(j)\right)-H_{k}(j)\right)^{2}}{2 \operatorname{Var}\left(\xi_{k}(j)\right)}\right) \\
& \exp \left(-\frac{\left(R(j)-E\left(\xi_{k}(j)\right)+H_{k}(j)\right)^{2}}{2 \operatorname{Var}\left(\xi_{k}(j)\right)}\right) \\
& =\left(\frac{2 H_{k}(j)\left(R(j)-E\left(\xi_{k}(j)\right)\right)}{\operatorname{Var}\left(\xi_{k}(j)\right)}\right) \forall k, j .
\end{aligned}
$$

In case of non-Gaussian approximation, $\xi_{\mathrm{k}}(\mathrm{j})$ can be approximated using three different non-Gaussian models as, alpha-stable, Laplace, and Gaussian mixture model. As Cauchy and Laplace models considered a good approximation for impulsive noise only (i.e. $\left.n(j)=n_{I}(j)\right), \xi_{k}(j)$ in (8) has either Cauchy or Laplace distribution, so we have:

$$
\begin{gathered}
E(R(j))=\sum_{k=1}^{K} H_{k}(j) E\left(x_{k}(j)\right) \\
\operatorname{Var}(R(j))=\sum_{k=1}^{K}\left|H_{k}(j)\right|^{2} \operatorname{Var}\left(x_{k}(j)\right)+\sigma_{I}^{2}
\end{gathered}
$$

Where $\sigma_{I}^{2}$ is the power of the impulsive noise occurs during the $j^{\text {th }}$ chip. While, GMM is considered a good fit for both Gaussian and non-Gaussian noise (i.e. $n(j)=n_{a}(j)+$ $\left.n_{I}(j)\right), \xi_{k}(j)$ has GMM distribution, hence from (8) we have:

$$
\begin{gathered}
E(R(j))=\sum_{k=1}^{K} H_{k}(j) E\left(x_{k}(j)\right) \\
\operatorname{Var}(R(j))=\sum_{k=1}^{K}\left|H_{k}(j)\right|^{2} \operatorname{Var}\left(x_{k}(j)\right)+\sigma^{2}+\sigma_{I}^{2} .
\end{gathered}
$$

For alpha stable distribution, with $\alpha=1$ (Cauchy detector). Therefore, the ESE outputs are the (LLRs) about $\left\{x_{k}(j)\right\}$ as follow [17]:

$$
\begin{aligned}
& L\left\{x_{k}(j)\right\} \\
& =\log \left[\frac{\gamma^{2}+\left(r(j)-E\left(\xi_{k}(j)\right)+h_{k}(j)\right)^{2}}{\gamma^{2}+\left(r(j)-E\left(\xi_{k}(j)\right)-h_{k}(j)\right)^{2}}\right] \forall k, j .
\end{aligned}
$$

The Cauchy detector has been used as a suboptimal detector to model the impulsive noise only with a robust performance, but it is still complex because of the need to calculate the log operation [17].

For Laplace distribution, the case in which the noise samples $\xi_{k}(j)$ have a Laplace distribution, the ESE outputs are the (LLRs) about $\left\{x_{k}(j)\right\}$ as follow:

$$
L\left\{x_{k}(j)\right\}=\frac{2}{b}\left(\begin{array}{l}
\left|\frac{r(j)}{2}-\frac{\left.E\left(\xi_{k}(j)\right)-h_{k}(j)\right)}{2}\right| \\
-\left|\frac{r(j)}{2}-\frac{\left.E\left(\xi_{k}(j)\right)+h_{k}(j)\right)}{2}\right|
\end{array}\right)
$$


This form is a sum over partial decision statistics which can be written as [4]:

$$
\begin{aligned}
& \left|\frac{r(j)}{2}-\frac{\left.E\left(\xi_{k}(j)\right)-h_{k}(j)\right)}{2}\right|-\left|\frac{r(j)}{2}-\frac{\left.E\left(\xi_{k}(j)\right)+h_{k}(j)\right)}{2}\right| \\
& =\left\{\begin{array}{l}
\left.\left.E\left(\xi_{k}(j)\right)-h_{k}(j)\right) r(j) \geq E\left(\xi_{k}(j)\right)-h_{k}(j)\right) \\
\left.\left.r(j) E\left(\xi_{k}(j)\right)+h_{k}(j)\right)<r(j)<E\left(\xi_{k}(j)\right)-h_{k}(j)\right) . \\
\left.E\left(\xi_{k}(j)+h_{k}(j)\right) r(j) \leq E\left(\xi_{k}(j)\right)+h_{k}(j)\right)
\end{array}\right.
\end{aligned}
$$

Laplace distribution has been used as a good approximation for impulsive noise also with complexity lower than Cauchy model since it needs to calculate the sum operation only.

For GMM distribution, the case in which the noise samples $\xi_{\mathrm{k}}(\mathrm{j})$ have a GMM distribution, the ESE outputs are the (LLRs) about $\left\{\mathrm{x}_{\mathrm{k}}(\mathrm{j})\right\}$ as follow [20]:

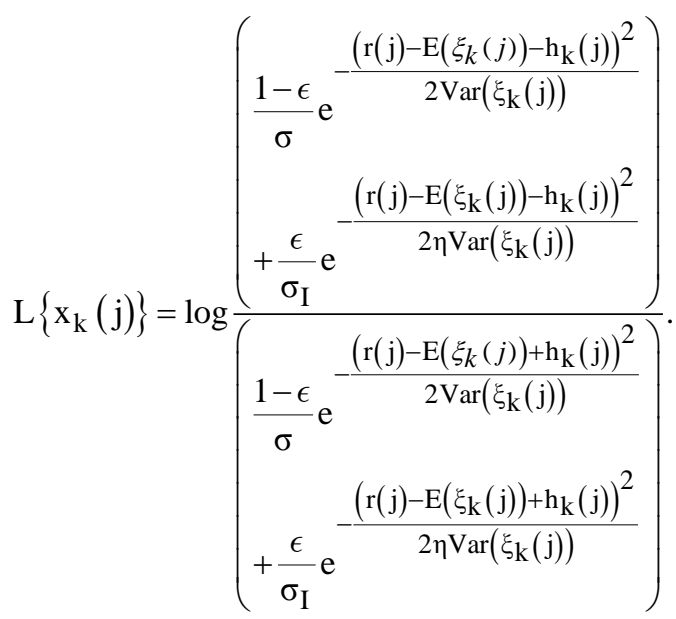

GMM is considered an excellent fit to simulation because it can adapt between AWGN and impulsive noise but with high complexity since many parameters and operations need to be estimated.

After the end of ESE process, The LLR-valued chip sequence $L\left(x_{k}(j)\right)$ is then de-interleaved to produce LLRs of $c_{k}(j),\left\{L\left(c_{k}(j)\right)\right\}$ which is delivered to DECs part discussed as follows.

\subsubsection{The DEC Function}

The DECs in Figure 1(b) consists of SPA of LDPC decoder and soft in soft out CBC spread/de-spread operations. The DECs output are the extrinsic LLRs of $c_{k}(j),\left\{\operatorname{Ext}\left(c_{k}(j)\right)\right\}$, which is interleaved to produce $\left\{\right.$ Ext $\left.\left(\mathrm{x}_{\mathrm{k}}(\mathrm{j})\right)\right\}$ and then fed back to the ESE. In the next iteration, $\left\{\operatorname{Ext}\left(\mathrm{x}_{\mathrm{k}}(\mathrm{j})\right)\right\}$ is used as a priori information to update $\mathrm{E}\left(\mathrm{x}_{\mathrm{k}}(\mathrm{j})\right)$ and $\operatorname{Var}\left(\mathrm{x}_{\mathrm{k}}(\mathrm{j})\right)$ as [13]:

$$
\begin{gathered}
E\left(x_{k}(j)\right)=\tanh \left(\frac{\operatorname{Ext}\left(x_{k}(j)\right)}{2}\right) \\
\operatorname{Var}\left(x_{k}(j)\right)=1-E\left(x_{k}(j)\right)^{2} .
\end{gathered}
$$

As discussed in Eq. (10), $\mathrm{E}\left(\mathrm{x}_{\mathrm{k}}(\mathrm{j})\right)$ and $\operatorname{Var}\left(\mathrm{x}_{\mathrm{k}}(\mathrm{j})\right)$ will be used in the ESE to update the interference mean and variance [8]. The effect of impulsive noise is regarded as interference. Due to random interleaving and de-interleaving process, the impulsive noise is spread out over the interleaving block. These interleavers disperse the resultant sequences so that the adjacent chips are approximately uncorrelated, which facilitates the simple chip-by-chip detection scheme. This iterative process is repeated a preset number of times. In the final iteration, the DECs produce a hard decision on information bit which is decoded by LDPC decoder. The iteration number can be adjusted depending on system requirement and computational power [13].

\section{Simulation Results}

In this section, the simulation results demonstrate the performance of LDPC-Coded OFDM-IDMA-UWB system. The discrete time channel model proposed by the IEEE 802.15.3a working group [14] is utilized, which is based on the modified $\mathrm{S}-\mathrm{V}$ model. This contribution focuses on the line-of-sight (LOS) channel model 1 (CM1) which corresponds to a short-range $(0-4 \mathrm{~m})$ indoor wireless environment. The simulation results are averaged over a large number of channel realizations using Matlab.

Not only AWGN is considered here but also non-Gaussian noise models like standard alpha stable, Laplacian, and GMM.

The simulation parameters are summarized as follows:

Regular $(128,256)$ LDPC codes are applied to 128 data length in system simulation whose parity-check matrix has 128 rows and 256 columns. Obviously, the corresponding coding rate is $1 / 2$. FFT size $=256$, and cyclic prefix $=32$ was added to each OFDM symbol block to avoid the effect of inter-chip interference. A common length-32 spreading sequence is assigned to all users, and a randomly generated chip interleaver is allocated to each user. The maximum iteration number of SPA decoding is 7 , and the Iteration number of IDMA receiver is 3 . The simulation is performed for (1-32) users. It is assumed that all users initially are synchronous with equal power allocation. The receiver is assumed to have perfect knowledge of the channel state information.

The simulations have three cases: The first case studies the BER performance of the proposed system for multi-users $(\mathrm{k}=1,8,16,32)$ under Gaussian and non-Gaussian noise models. The second case presents the performance comparison of the proposed LDPC-coded OFDM-IDMA for eight users under the studied noise models. Finally, the last case investigates the performance comparison between un-coded and LDPC-coded OFDM-IDMA UWB systems for eight users under the studied noise model.

Figure 2 - Figure 5 show the performance of the proposed LDPC-coded OFDM-IDMA-UWB system for multi users $(k=1,8,16,32)$ under the Gaussian and nonGaussian noisy channel effect.

Figure 2 demonstrates the BER performance of the proposed system for multi-users under the effect of AWGN model. The results indicate the power of LDPC coding technique on system performance. It can be seen that the proposed system can achieve near single user performance for 16-users and the performance is degraded by about $1.3 \mathrm{~dB}$ for 32-users compared with single user.

Figure 3 shows the BER performance of the proposed system for multi-users under Cauchy noise model ( $\propto=1$ in alpha stable distribution ). The results show that the system achieves a significant improvement under this model for all users with minor degradation by about $1 \mathrm{~dB}$ compared with AWGN model. 


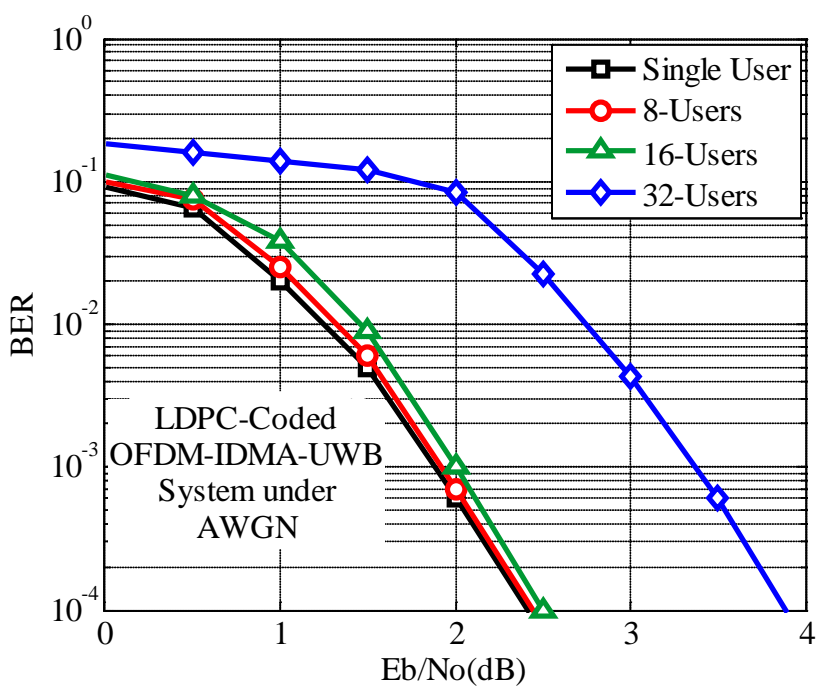

Figure 2. BER performance of LDPC-Coded OFDM-IDMA-UWB system for multi-users under AWGN

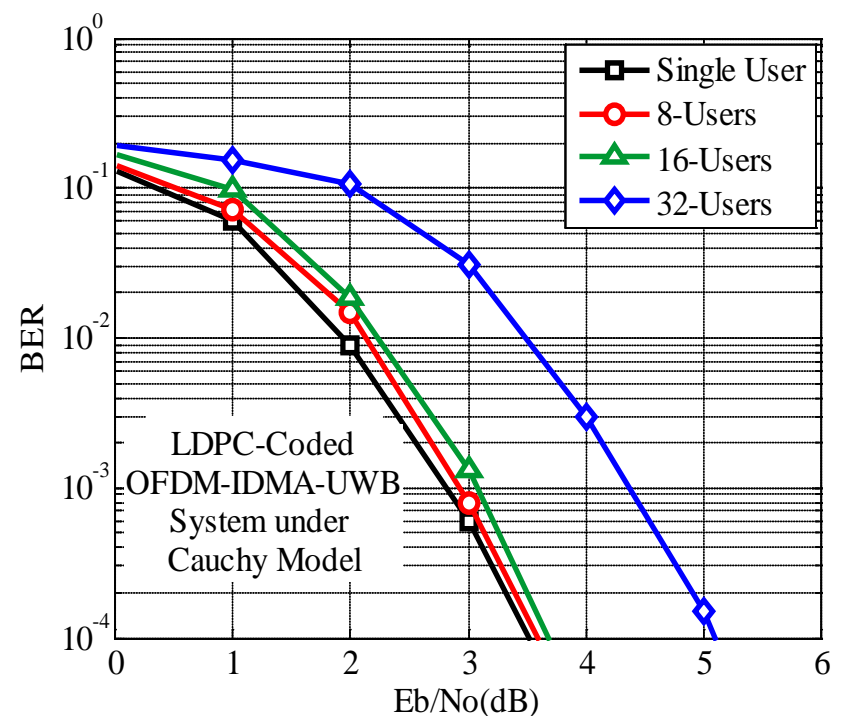

Figure 3. BER performance of the proposed LDPC-coded OFDMIDMA-UWB system for multi-users under Alpha-Stable (Cauchy) Model

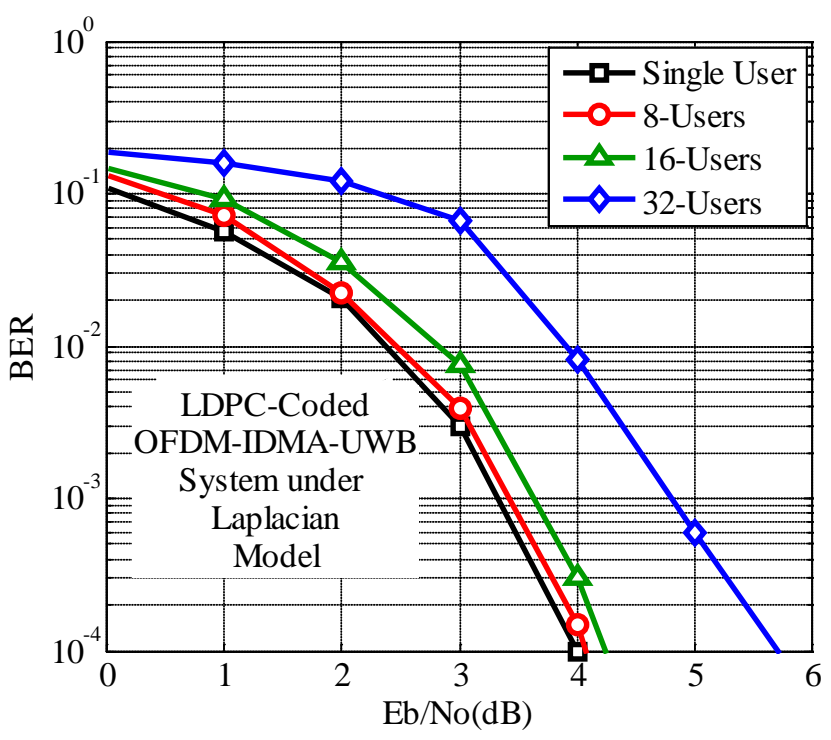

Figure 4. BER performance of LDPC-Coded OFDM-IDMA-UWB system for multi-users under Laplacian Model
Figure 4 shows the BER performance of the proposed system for multi-users under the effect of Laplacian noise model. We take a scale parameter $\mathrm{C}=1$ which is commonly used to represent the tail of impulsive noise. The results indicate that the BER performance is degraded by about 1.5dB compared with AWGN model.

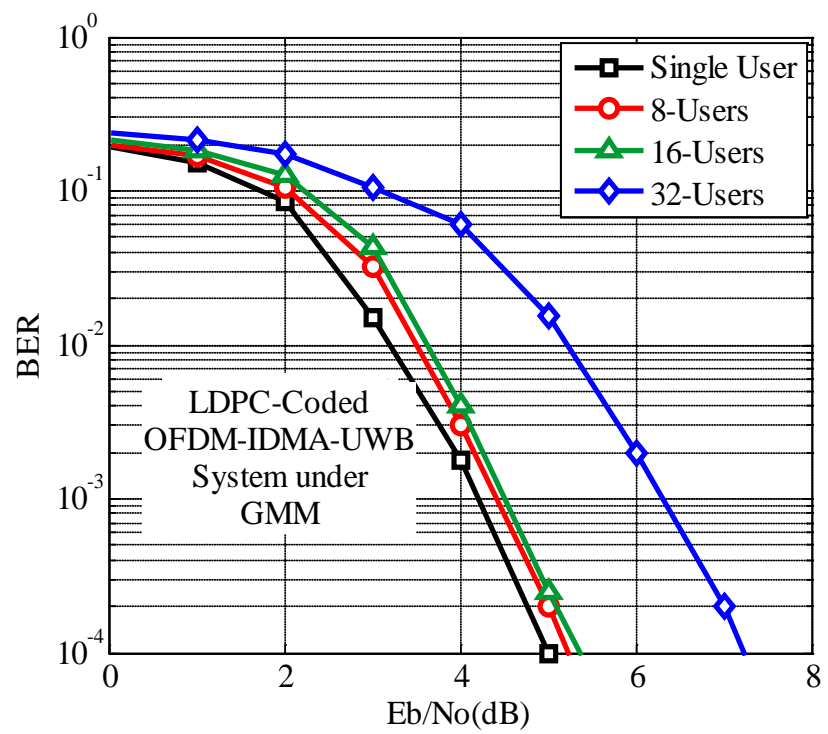

Figure 5. BER performance of Polar-Coded OFDM-IDMA-UWB system for multi-users under GMM

Figure 5 shows the BER performance of the proposed system for multi-users under GMM effect. The most common values of impulsive and control parameters for GMM are $(\eta=10, \epsilon=0.1)$ respectively. It is observed that the performance is degraded by $2.5 \mathrm{~dB}$ w.r.to AWGN model.

From the previous study, BER performance comparison for 16 users under Gaussian and non- Gaussian noise model is introduced in Figure 6.

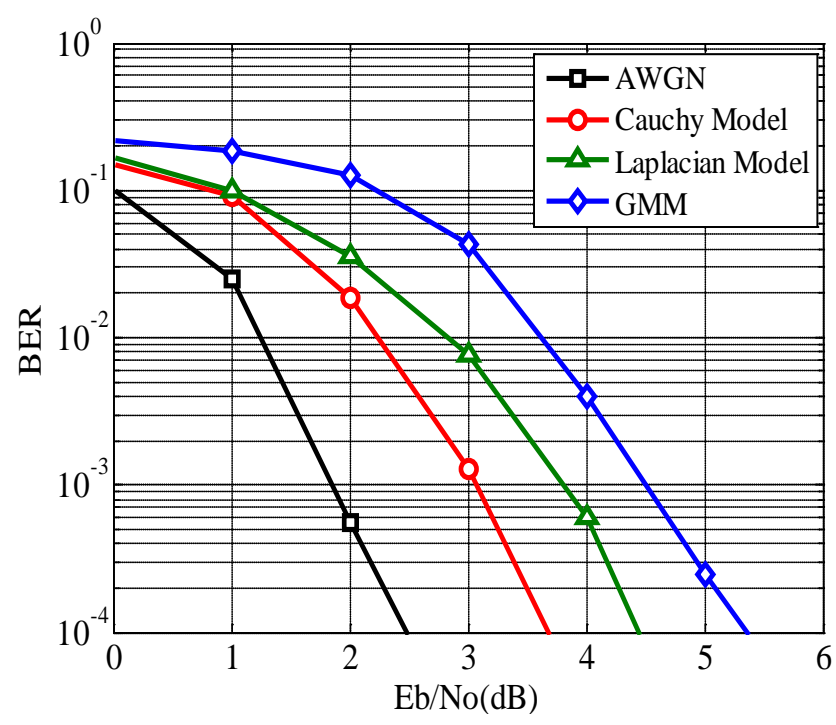

Figure 6. BER performance of LDPC-coded OFDM-IDMA-UWB system for 16-users under Gaussian and non-Gaussian noise models

The comparison clarifies that Cauchy model outperforms well than other non-Gaussian models and achieves good BER performance compared with AWGN model as demonstrated in Table 1. 
Table 1. Performance Comparison of LDPC- IDMA-OFDM-UWB System under four Noise Models

\begin{tabular}{|c|c|c|c|c|}
\hline \multirow{2}{*}{ LDPC-IDMA-OFDM-UWB System } & \multirow{2}{*}{ No. of Users (K) } & \multirow{2}{*}{ Spread length (Sp) } & Noise Model & $\begin{array}{c}\left(\frac{E_{b}}{N_{o}} @ B E R=10^{-4}\right) \\
d B\end{array}$ \\
\hline \multirow{2}{*}{ CM1, data length N=128 bit, rate=1/2 } & \multirow{2}{*}{16} & \multirow{2}{*}{32} & AWGN & 2.5 \\
\cline { 3 - 5 } & & & Cauchy & 3.75 \\
\cline { 3 - 5 } & & & Laplace & 4.3 \\
\hline
\end{tabular}

Table 2. Performance Comparison between Un-Coded and LDPC-Coded IDMA-OFDM-UWB Systems

\begin{tabular}{|c|c|c|}
\hline \multicolumn{2}{|c|}{$\mathrm{E}_{\mathrm{b}} / \mathrm{N}_{0}(\mathrm{~dB})$ at BER $=10^{-4}$} \\
\hline System Performance & Un-Coded IDMA-OFDM-UWB System & LDPC-Coded IDMA-OFDM-UWB System \\
\hline Noise Models & 9 & 3.75 \\
Cauchy & 10 & 4.3 \\
Laplacian & 11 & 5.25 \\
GMM & & \\
\hline
\end{tabular}

But on the other hand, GMM can be considered an excellent fit to simulation because it can adapt between AWGN and impulsive noise but with high complexity, compared with Cauchy and Laplacian models, since many parameters and operations need to be estimated.

Finally, a comparative analysis between the proposed LDPC-coded and un-coded OFDM-IDMA-UWB systems under the studied noise models is introduced in Figure 7.

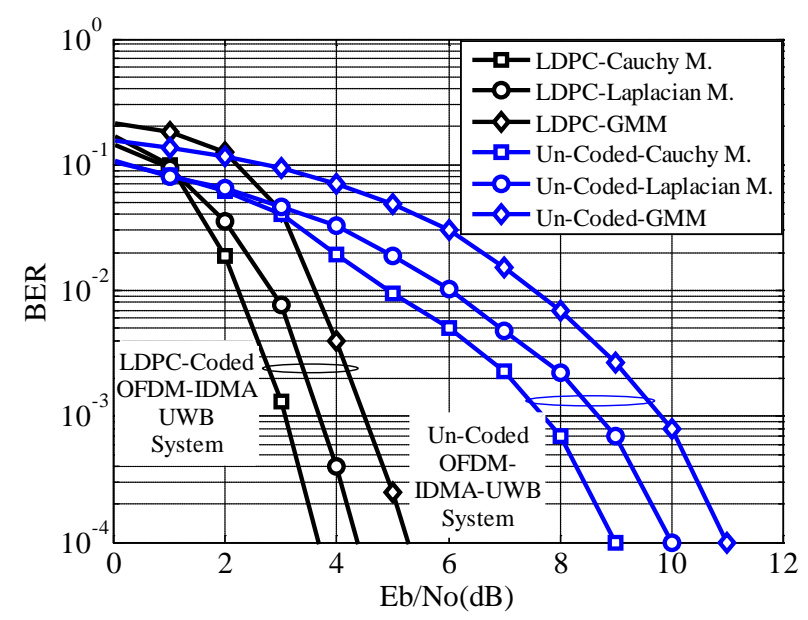

Figure 7. BER performance comparison between un-coded and LDPCcoded OFDM-IDMA-UWB systems for 16-users under non-Gaussian models

The simulation results indicate that the BER performance of LDPC-coded system is improved for 16-users compared with un-coded system under non-Gaussian models as demonstrated in Table 2.

These results are roughly similar to the results obtained in [10] but instead of applying non-Gaussian approximation for the system interferences and employing LDPC coding technique to improve the system performance, the author in [10] proposed different techniques such as hard and soft narrowband interference notching and a joint power and subcarrier allocation. Also, this work can be compared with the proposed system in [13] which gives nearly the same BER performance under AWGN model.

\section{Conclusion}

This paper addresses the performance of the proposed LDPC-coded OFDM-IDMA-UWB system over Gaussian and non-Gaussian noisy channels. Three different noise models, standard alpha stable, Laplacian, and GMM, have been introduced to represent the non-Gaussian noise which are more realistic for UWB systems.

LDPC code is merged with IDMA scheme based OFDM system to overcome the multipath channel imperfections efficiently and improve the performance.

The performance evaluation of the proposed system have been introduced and then compared with un-coded OFDM-IDMA-UWB system under the studied noise models. The simulation results show that, not only the proposed LDPC-coded OFDM-IDMA-UWB system has an efficient performance improvement compared with uncoded OFDM-IDMA-UWB system, but also the proposed system achieves near single user performance under the studied noise models with low receiver complexity.

\section{References}

[1] Hussein Hijazi and Laurent Ros, "Rayleigh Time-varying Channel Complex Gains Estimation and ICI Cancellation in OFDM Systems," European Transactions on Telecommunications, April 2009.

[2] Montojo-Bennassar, Juan I., "Adaptive OFDM-based UWB," Ph. D., UC San Diego Electronic Theses and Dissertations, 2009.

[3] Haleh Hosseini, and Rozeha bt. A. Rashid, "Study on UWB Systems in the Presence of Narrowband Interference," IEEE Proceedings of 2008 Student Conference on Research and Development, Malaysia, 2008.

[4] N. C. Beaulieu and David J. Young, "Designing Time-Hopping Ultra-Wide Bandwidth Receivers for Multi-User Interference Environments,” in Proc. IEEE Int. Conf. Commun., Beijing, May 2008.

[5] B. Hu and N. C. Beaulieu, "On characterizing multiple access interference in TH-UWB systems with impulsive noise models," in Proc. IEEE Radio andWireless Symp., Orlando, FL, Jan. 2008, pp. 879-882.

[6] Li Ping, "Interleave Division Multiple Access and Chip By Chip Iterative Multi User Detection," IEEE Communication Magazine, vol. 43(6), pp. S19-S23, June 2005.

[7] L. Ping, L. Liu, K. Y. Wu, and W. K. Leung, "Interleave-division multiple-access,”IEEE Trans. Wireless Commun., vol. 5, no.4, pp. 938-947, Apr. 2006.

[8] Vishal Shukla, M. Kumar Shukla, Tanuja Pande, "Multiuser Detection using IDMA Scheme in UWB Home Environment," International Journal of Computer Applications (0975-8887) Volume 55-No.13, October 2012.

[9] Rong Zhang and Lajos Hanzo, "Iteratively Detected Multi-Carrier Interleave Division Multiple Access," MICROCOLL'07, BUDAPEST, HUNGARY, MAY 2007.

[10] Kai Yang, and Xiaodong Wang, "A Multicarrier InterleaveDivision UWB System," IEEE Transactions on Wireless Communications, VOL. 5, NO. 7, JULY 2006. 
[11] J. Ling, L. Wang, Y. Luo, H. Wang, "Performance of LDPCCoded Interleave-Division Multiple-Access (IDMA) System,” IET ICWMMN Proceedings. Hangzhou, China, Nov. 2006.

[12] Ajit Singh and Rajan Mishra, "Design of IDMA Scheme Using LDPC Coding", VSRD-IJEECE, Vol. 1 (7), 2011, 364-373.

[13] Wei-Chieh Huang, Kuo-Sheng Lu, Chih-Peng Li, and Hsueh-Jyh $\mathrm{Li}$, "Performance Evaluation for LDPC Coded OFDMIDMASystems over Frequency Selective Fading Channels," IEEE Transactions on Wireless Communications, August 2010.

[14] AA. Saleh, RA.Valenzuela, "A statistical model for indoor multipath propagation," IEEE Journal on Selected Areas Communication, vol.5, no.2, pp.128-37, 1987.

[15] Xiang Chen, Fengzhong Qu and Liuqing Yang, "OFDM-IDMA for power line communication," IEEE International Symposium on Power Line Communications and Its Applications, April 2011.

[16] Hamza El Ghannudi, Laurent Clavier, Nourddine Azzaoui, Fran_cois Septier, Paul-Alain Rolland, "Alpha-stable interference modeling and Cauchy receiver for an IR-UWB ad hoc network", HAL archieves, HAL Id: hal-00685924, April 2012.

[17] Tarik Sayed, "Receiver Design for Signals in Non-Gaussian Noise Applications to Symmetric Alpha-Stable and Middleton's Class-A Noise Models", phD thesis, Carleton University Ottawa, Ontario, Canada, K1S 5B6,June 2012.

[18] Yaping Bao, Yingdong Zhu, Xiaomei Zhu, and Yuzhi Chu,
"A spectrum sensing method based on fractional lower order moments in weakly correlated Laplace noise", Proceedings of APSIPA Annual Summit and Conference 2015.

[19] Nazl Guney, Hakan Delic, Mutlu Koca, "Robust Detection of Ultra-Wideband Signals in Non-Gaussian Noise," IEEE Transaction on microwave theory and techniques, Vol. 54, No. 4, April 2006.

[20] Arun Ayyar, Michael Lentmaier, K. Giridhar and Gerhard Fettweis, "Robust Initial LLRs for Iterative Decoders in Presence of Non-Gaussian Noise", IEEE International Symposium on Information theory, 2009.

[21] Mr. Paurush Bhulania, Mr. Gagan Minocha, Ms. Richa Sharma, "Issues in Analysis \& Design of IDMA \& its Application", International Journal of Advanced Research in Electrical, Electronics and Instrumentation Engineering, Vol. 2, Issue 5, May 2013.

[22] Zhen-hua WANG, Ge-fei YU1, Xi CHENG, Jie Yang, Yin-ting WANG, "Study on Simulation Performance of OFDM-IDMA System", Proceedings of the 2nd International Conference on Computer Science and Electronics Engineering (ICCSEE 2013).

[23] A. Alagulakshmi, V. Arun, "Performance Improvement of OFDMIDMA with Modified SISO", International Journal of Research in Electronics \& Communication Technology Volume-2, Issue-2, IASTER 2014. 